\title{
Comment on "In situ multielemental monitoring in coral skeleton by CF-LIBS" by S. Pandhija and A.K. Rai (DOI:10.1007/s00340-008-3343-5)
}

\author{
G. Nehrke • G. Langer
}

Received: 12 May 2011 / Published online: 29 July 2011

(C) Springer-Verlag 2011

The authors state that they investigated the elemental composition of a coral skeleton, but the image they present (Fig. 2) displays barnacles (attached to a piece of rock), belonging to the Arthropoda. Corals, although being animals as well, belong to the Cnidaria. These two groups of organisms, Arthropoda and Cnidaria, are clearly phylogenetically separated and have no morphological features in common. Some corals represent reef building organisms which can lead to the formation of massive structures extending across several kilometers, whereas barnacles are objects of a few centimeters in size. The authors describe corals to be among the oldest and most primitive sea organisms. Both statements are not correct. The term "primitive" should not be used in an evolutionary context and corals do not belong to the oldest marine organisms. Corals appeared ca. 550 million years BP, whereas cyanobacteria have evolved ca. three billion years ago.

We think that it is not only necessary to point out that the authors did not do the measurements on the material they thought they would, but also to comment on two crucial statements from the introduction and conclusion section.

As objective of the paper the following is stated at the end of the introduction section:

"The primary objective of this study is to estimate the concentrations of elements present in the coral skeleton using CF-LIBS approach. This analysis is significant in view of the fact that corals have become an important source of $\mathrm{Sr}$ and that a coral implanted on bone tissue is gradually transformed to become a neoformed bone."

Here we would like to point out that the statement that "corals have become an important source of $\mathrm{Sr}$ " is not backed up by a reference and seems to be most unlikely since $\mathrm{Sr}$, as opposed to $\mathrm{Ca}$, is a minor element in the coral skeleton (and depleted compared to its concentration in seawater). Moreover, it is unclear why the usage of corals in bone crafting should benefit from knowing the concentration of $\mathrm{Sr}$ in the coral skeleton.

In the conclusion it is stated that:

"The present study suggests that coral skeleton can be an important source of some essential elements like $\mathrm{Sr}$, $\mathrm{Ca}$, etc. that are used in natural therapies, for treatment of osteoporosis and in strengthening bone growth."

Apart from the fact that "natural therapies" is a very vague and undefined expression it is not correct that knowing the elemental composition of a coral skeleton can improve the treatment of serious diseases. The authors demonstrated that CF-LIBS measurements can be used to measure the elemental composition of the material investigated. The composition of the investigated material on the other hand had already been determined using several other methods, and clinical studies on the treatment of osteoporosis and other diseases have not been performed by the authors. 